\title{
A Crictal Review of Cryptocurrency Systems
}

\author{
Article by Mahendra Kumar Shrivas ${ }^{1}$, Thomas Yeboah ${ }^{2}$ \\ ${ }^{1}$ Information Technology, Texila American University, Guyana, South America \\ ${ }^{2}$ Department of ICT, Christen Service University College, Kumasi, Ghana \\ E-mail:mshrivas@texilaconnect.com ${ }^{1}$ thomyebs24@gmail.com ${ }^{2}$
}

\begin{abstract}
Adoption of Cryptocurrency has grown significantly over the time and becoming more popular among young generation. People are calling it currency of new digital era. In this research work we are reviewing the dominant Cryptocurrency systems and its underlying disruptive Innovations and Technologies.
\end{abstract}

Keywords: Cryptocurrency, Blockchain, Digital Currency, Hashing, Proof of Work, Proof of Stake, Tangle, RippleNet, Bitcoin, Altcoins.

\section{Introduction}

We are in the middle of next revolution where Innovations and Technologies are challenging our existing systems and disrupting the way we do several operations. From industrial revolution to the invent of Internet we have already seen lots of changes which have significantly affected our daily life. In today's era we cannot imagine our life without Internet. Our various industries are getting benefits from Smart Phones, Cloud Computing, Artificial Intelligence, Machine Learning, Big Data, Internet of Things (IoT), etc. These technologies are just not replacing the old technologies but also transforming our economy to more digitized and innovation driven economy. New set of services have been build on these technologies like eBanking, eLearning, eCommerce, eGovernance, etc.

Cryptocurrency is an example of such disruptive Innovation and Technology which is transforming the way we do banking in this globalized world which is powered by Internet.

A Cryptocurrency is cryptographically signed digital currency which is virtual in nature and hard to counterfeit due to strong encryption. Cryptocurrency is different from fiat currency. Fait currencies are printed by various governments and they control the value of it while governments are not having any control over Cryptocurrency and mostly decentralized. Crypto-currencies are controlled by Cryptocurrency community members, miners, general public participating in the transaction. Transactions are processed, validated, verified by Cryptocurrency miners and successful transaction get recorded in public and distributed ledger called Blockchain. Cryptocurrency transactions are anonymous, irreversible and secure in nature.

\section{A. Objectives of the study}

The main objectives of this Study is to:

- Critically review the underline technologies being used in dominant Cryptocurrency Systems.

- Analyze strengths and weaknesses of underline technologies of dominant Cryptocurrency Systems.

\section{B. Significance of the study}

Cryptocurrency has been adopted significantly by various financial insinuations. Various merchants have started accepting Crypto-currencies along with fiat currencies. Cryptocurrency has given new opportunity to governments, businesses, individuals, start-ups to build future ready, open, reliable and secure services which could be helpful to rebuild dying global economy. This study could be a significant contribution in literature on Technologies of Cryptocurrency Systems as authors are going to review technologies being used in dominant Cryptocurrency Systems and also going to discuss advantages and disadvantages of Cryptocurrency. 
DOI: $10.21522 /$ TIJAR.2014.04.02.Art012

ISSN: $2520-3088$

\section{Scope of the study}

In this research work authors are going to review underline technologies of dominant Cryptocurrency Systems as per as Innovation and Technology is concern which is not linked with market capitalization, valuation or legal status of Cryptocurrency.

\section{Research methodology}

In this study authors have used Qualitative research methodology. Mainly Exploratory and Descriptive approach adopted in this critical review and based on observation.

\section{Literature review}

Wei Dai published a description of "b-money", a distributed and an anonymous electronic cash system in 1998 (Dai, 2017). As per Wei Dai "b-money, a scheme for a group of untraceable digital pseudonyms to pay each other with money and to enforce contracts amongst themselves without outside help". Later on Nick Szabo created "bit gold" (Peck, 2012). Bit Gold was a Cryptocurrency and was based on Proof of Work (POW) function (Szabo, 2008). Reusable Proof of Work ("RPOW") was proposed by Hal Finney in which was an extension to Dai's and Nick's work. The RPOW system reuses POW tokens and uses Hash-Cash (Finney, 2005). Hash Cash was based on SHA-1 hashes (Eastlake 3rd \& Jones, 2001). At this time none of the Cryptocurrency become famous.

And then the global financial crisis begins in 2007 when subprime lending market in US breakdown due high debt risk Lehman Brothers collapsed on 15th September 2008 (Williams, 2010). 2007 and 2008 was the year of global recession, economy slowdown and debt crisis. Double spending was the fundamental issue which leads to this financial crisis along with mismanagement of funds by various financial institution and governments.

In 2009 a purely peer-to-peer, decentralized, electronic cash called Bitcoin was introduced by pseudonym Satoshi Nakamoto which could be an individual or group (Nakamoto, 2008). Bitcoin was the first Cryptocurrency which become famous among general public. Since then lots of similar Cryptocurrency was introduced with similar or new technology and known as Altcoins.

Later in 2011 the Financial Crisis Inquiry Commission (FCIC) (FCIC, 2017) given reason behind this financial tragedy. As per the FCIC the financial disaster was preventable and was mainly caused by "widespread failures in financial regulation and supervision", "dramatic failures of corporate governance and risk management at many systemically important financial institutions", "a combination of excessive borrowing, risky investments, and lack of transparency" by financial institutions and what "added to the uncertainty and panic" was, hostile groundwork and fickle act by government (FCIC, 2011).

This fueled the adoption and technical enhancement of Cryptocurrency. Followings are the underline technologies being used in dominant Cryptocurrency Systems: -

\section{A. Blockchain}

Blockchain is one of widely used non-controversial technology in Cryptocurrency space which works flawlessly, securely and is a most valued technology after Internet. Blockchain maintains a public ledger which is a central but distributed record of all successful event/transaction in Blockchain networks. Once transaction is recorded in Blockchain it is impossible to remove or reverse. Verification of each transaction in this public ledger is done by agreements of a mainstream of the contributors in the Blockchain (Michael, Pattanayak, Verma, \& Kalyanaraman, 2016). Block ownership in Blockchain can be verified thus forged entry can be avoided. 


\section{Transaction in blockchain}

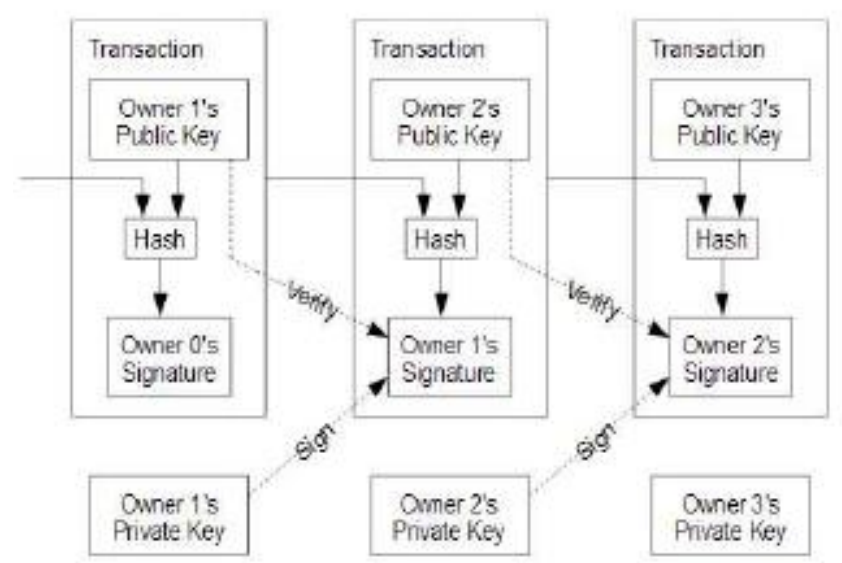

Figure 1. Transaction in blockchain

As depicted in Figure 1 (Nakamoto, 2008) in Blockchain each transaction has chain of cryptographically signed digital signatures which is irreversible. Each party transmit the digital information to the next party by digitally signing a hash of the prior transaction and the public key of the subsequent owner and adding these to the end of the digital information. Any changes in the block leads to different hash thus the changed block is dropped to avoid forging. Timestamps are hashed to track that data was existing already before it is processed by next node in the Blockchain network. High level and simplified version of Blockchain transaction is given in Figure 2 (Michael, Pattanayak, Verma, \& Kalyanaraman, 2016)

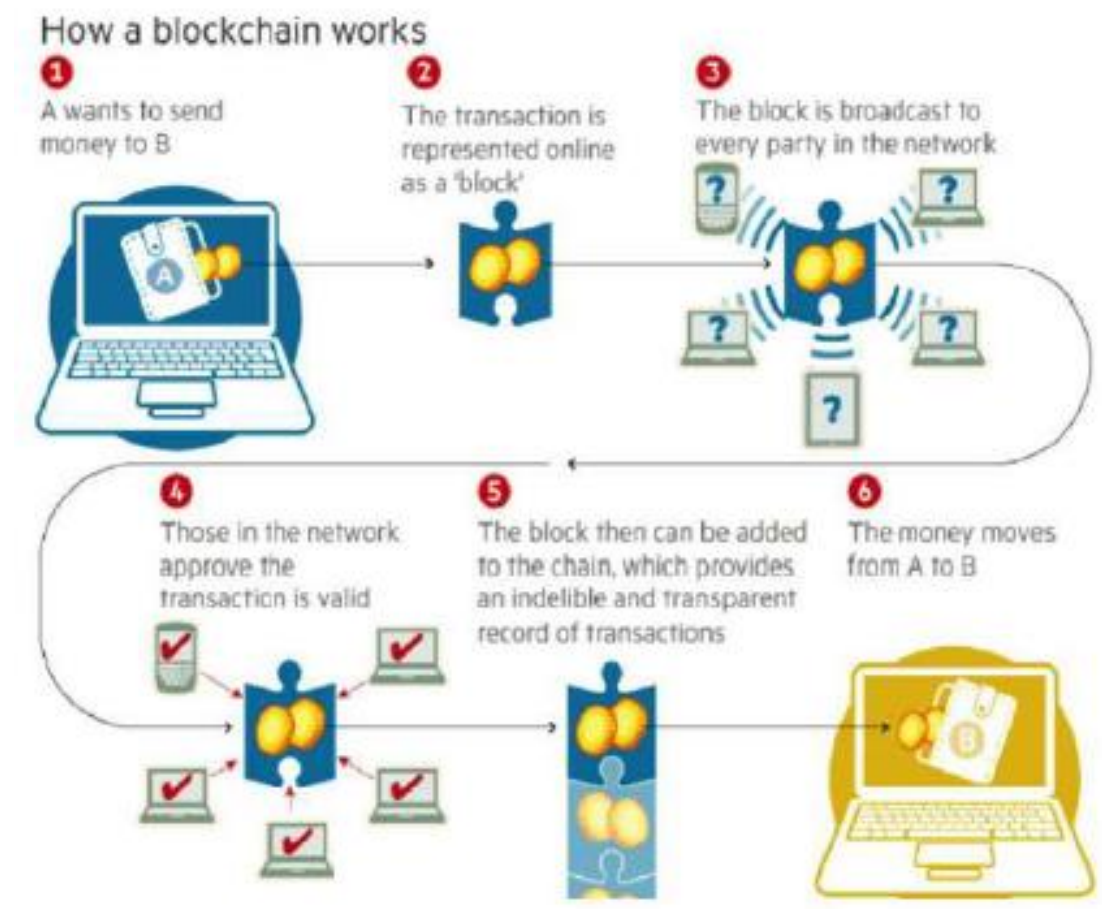

Figure 2. High level bitcoin transaction in blockchain

\section{Proof of work}

Proof of work (POW) concepts was introduced in "Bit Gold" later on it has been used in Hashcash as Reusable Proof of Work (RPOW) was introduced in 1998. In 2002 Adam Back introduced the concepts of CPU cost function which can be used as proof-of-work. Costs functions can be interactive 
DOI: $10.21522 /$ TIJAR.2014.04.02.Art012

ISSN: $2520-3088$

and non-interactive which can be used in connection oriented and connectionless environment respectively (Back, 2002).

As per Satoshi Nakamoto "The proof-of-work involves scanning for a value that when hashed, such as with SHA-256, the hash begins with a number of zero bits. The average work required is exponential in the number of zero bits required and can be verified by executing a single hash." (Nakamoto, 2008).

Cryptocurrency protocol uses Proof of Work to prevent double spending and to establish scarcity (Becker, et al., 2013).

\section{Incentive or mining rewards}

To keep Blockchain network running someone has to verify the transaction which involve execution of hashing algorithms and require significant CPU time and electricity resources. To reward supporting node in Blockchain network new coin is generated and given as a rewards to the supporting note which is also called miner. The incentive or mining rewards can also be given as transaction fees. Some of the Cryptocurrency which uses Blockchain as their fundamental underlying technology is listed in Table 1. However, they are having few difference in term of validation times, way of processing and services provided. Like Bitcoin and Bitcoin Cash is identical but uses difference block size. Bitcoin Cash uses higher block size and faster than Bitcoin. Ethereum and Dash is based on Virtual Machine but Dash uses two category of nodes one is master node and another one is validation node. While Ethereum is a decentralized Blockchain Application platform that runs smart contracts. Ethereum allows developers to design and issue their own Cryptocurrency. Ether is the fuel in Ethereum platform. Litecoin is similar to Bitcoin but is faster than Bitcoin as it takes 2.5-minute lesser block generation time. It uses scrypt hashing algorithm (Percival \& Josefsson, 2016) while Bitcoin uses SHA-256 hashing algorithm (Dadda, Macchetti, \& Owen, 2004). Litecoin uses Segregated Witness, and the Lightning Network technology to boost transaction in the Litecoin Blockchain network.

Some of the Strength of Blockchain is listed below:-

1. Blockchain is based on Peer-To-Peer concepts thus there isn't any third party or central bank sitting between sender and receiver.

2. Same currency cannot be spend at the same time.

3. Transactions are recorded in single public ledger and cannot be manipulated one recorded.

4. In traditional banking International transaction takes days to settle while in Blockchain it is a matter of few minutes.

5. Blockchain eliminates need of third-parties thus transaction fees are very low.

6. Users are empowered and having control of all their information and transactions.

7. Government cannot control or regulate Blockchain because users are allowed to do transaction anonymously.

8. Blockchain work on the concepts of decentralization thus Blockchain network cannot be closed down and users can do secure transaction 24/7.

9. Blockchain completely elements associated risks of frauds and identity thefts which is biggest issue in traditional banking system.

10. Blockchain tolerate unauthorized transaction and prevent malicious attacks. Some of the weaknesses of Blockchain is listed below:-

- Blockchain is power hungry. To keep Blockchain network running huge amount of power is needed. As per John McAfee, to generate 1 Bitcoin around 1000\$ get burnt.

- Various government regulation status is not clear on Blockchain. Legal adoption is an issue as per as Blockchain for Cryptocurrency is concern.

- Blockchain related technologies are still in its evaluation phase thus cyber security is an major issue.

- Due to anonymously nature of transaction this can be used by various criminals.

- To Integrate Blockchain to existing financial and banking system lots of extra capital and cost is required. 
- Customer protection is another issue in Blockchain, once transaction is committed, it cannot be reversed, unless the will of the new owner of the token.

- Increasing block size is the biggest challenge in Blockchain.

- Online Initial Coin Offering (ICO) frauds are common in Blockchain space so one should be very careful. Due to unclear regularity framework future of Blockchain related projects are in high risk. 


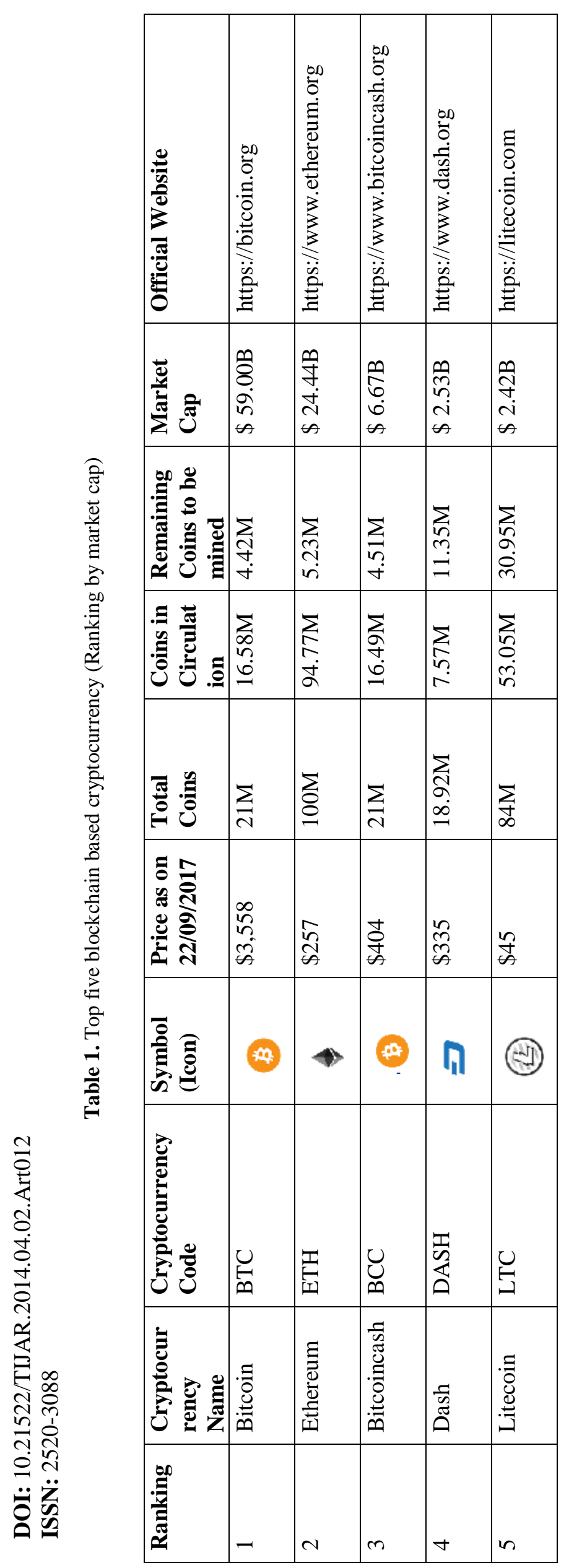




\section{B. RippleNet}

RippleNet is one connected global payment decentralized network which is based on Ripple and Network participants. AS Ripple.com "RippleNet is the world's only enterprise Blockchain solution for global payments." but it is different than other decentralized Cryptocurrency which uses Blockchain listed above. It's complete architecture is similar to current traditional Banking System where funds are being transferred between banks and MasterCard, Visa, Western Union, etc. verify transaction. So what is the different in RippleNet? In simple manner if we replace MasterCard, Visa, Western Union, etc. to Ripple with its xCurrent enterprise software that form RippleNet. So the next question is why banks are switching to RippleNet? Because it is fast, secure, less transaction fees and most important thing is that it can be regulated. Transaction in RippleNet not totally anonymous it maintains the record in Interledger along with other user information such as account and bank information. Each transaction in Interledger is verifiable along with other details. The whole RippleNet ecosystem members have been categories into two category. First is Network Members who are the enablers of RippleNet which includes Banks and Payment Providers and another one is Network Users which includes Platform Business, Corporate treasury, Banks, Payment Providers and Consumers. xCurrent is Ripple's enterprise software solution (Ripple.com, N.D.) which is used to do cross-border payment processing, end-to-end tracking and support two way information passing in RippleNet. In Figure 4 FX Ticker in xCurrent facilitate exchange between ledger and provide exchange rates. RippleNet is powered by the Interledger Protocol (ILP) (Thomas \& Schwartz, 2015) which enable to send payments between diverse ledger. xCurrent maintain ILP ledger. It is a sub-ledger of transacting bank's universal ledger. Validator in xCurrent is used to verify success and failure of payment transfer cryptographically. Ripple is the digital token which is used in RippleNet. Ripple related information has been given in Table 2. Ripple uses HTTPS protocol for communication link between bank and xCurrent. It uses OAuth 2.0 for authentication. Communication link within xCurrent component is also protected using HTTPS protocol (Ripple.com, N.D.).
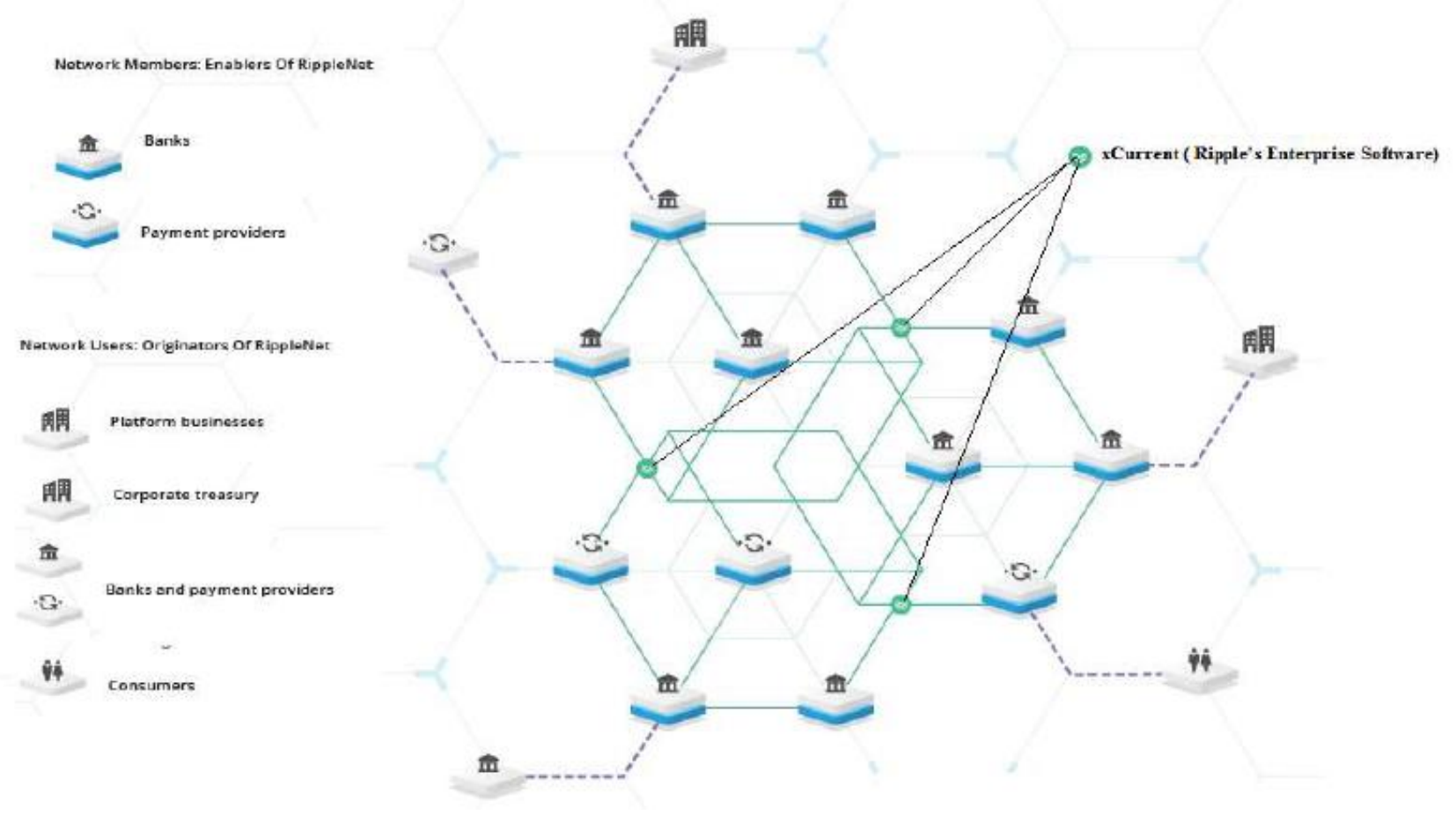

Figure 3. Ripplenet ecosystem 
DOI: $10.21522 /$ TIJAR.2014.04.02.Art012

ISSN: $2520-3088$

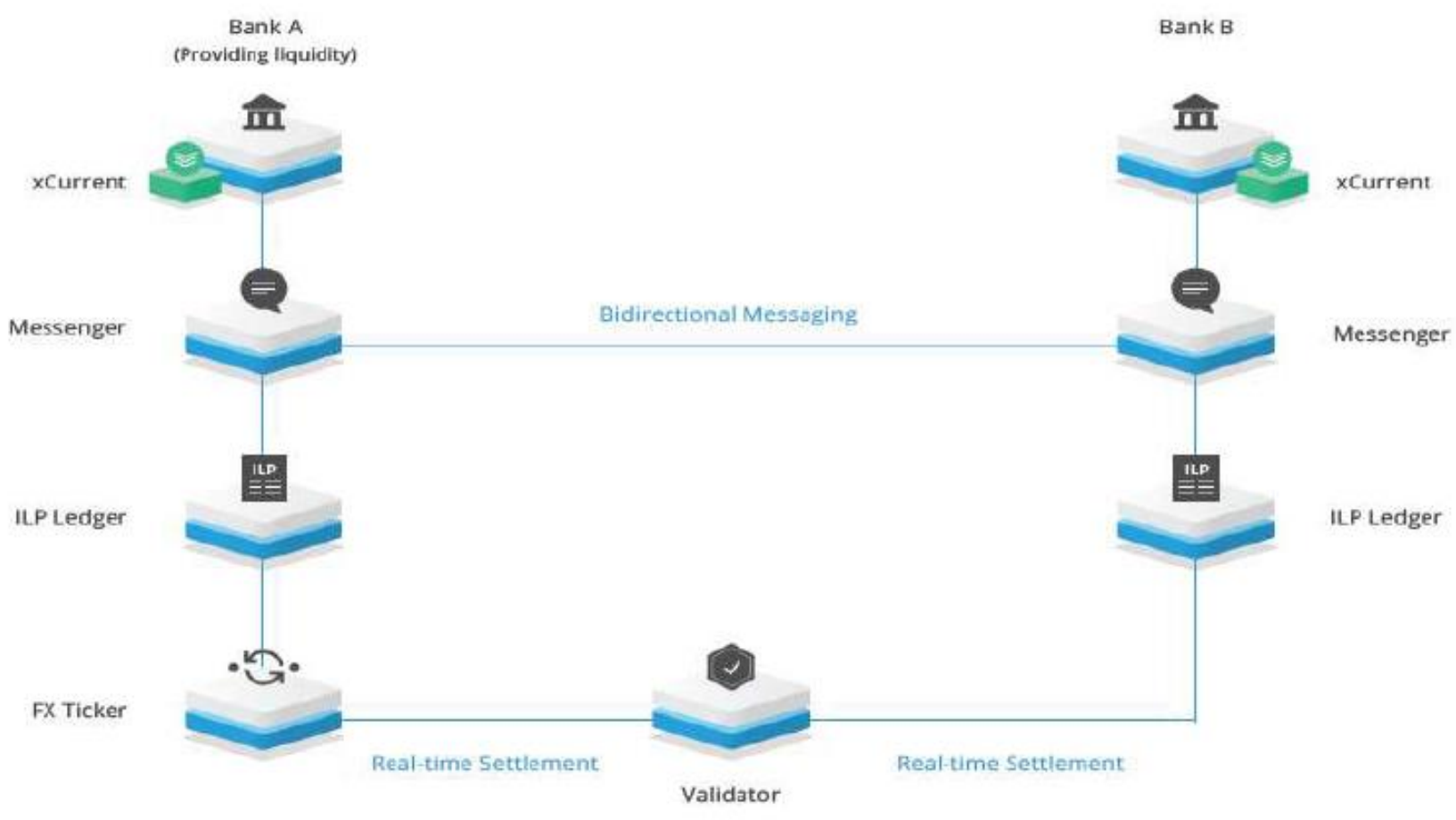

Figure 4. Ripplenet's xcurrent components

Some of the Strength of RippleNet Solution is listed below:

1. Certainty: Secure audible payments with settlement certainty.

2. Speed: Instant, on-demand and payment settlement 4 seconds

3. Scalable: RippleNet handles 1500 Transaction Per Second (TPS) and can be scaled to handled more transaction same as Visa which is currently handling around 50,000 TPS.

4. Cost: Low operational, liquidity costs, negligible energy consumption as compare to other Cryptocurrency.

5. Access: One stop solution and single point of access to an international network.

6. Compliance: Ripple provides licensed Solution to Banks and respects banks compliance responsibility. Thus regulation is not an issue with Ripple. More than 90 Banks/ Financial Institutions have already adopted or going to adopted RippleNet solution. Some of them are Banks like Bank of America, Standard Chartered Bank, Yes Bank, UBS, UniCredit, Axis Bank, AKBANK, SBI, etc. 
들 곻

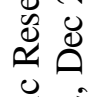

을

毅

.

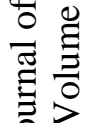

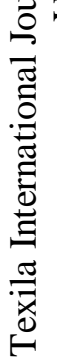

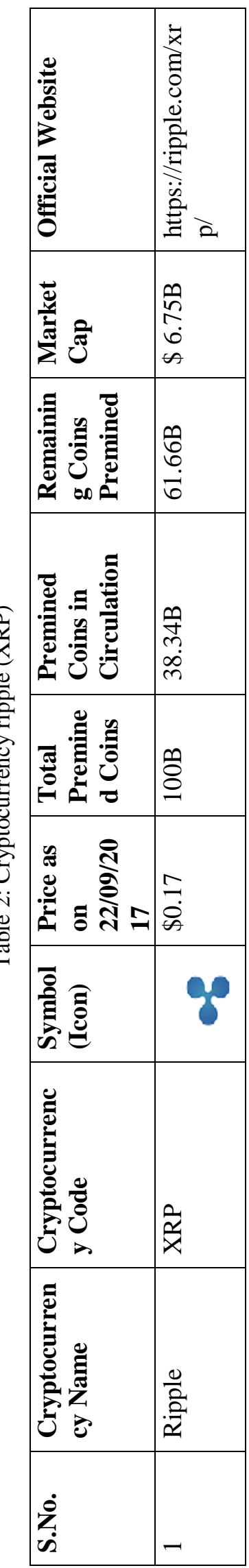


DOI: $10.21522 /$ TIJAR.2014.04.02.Art012

ISSN: $2520-3088$

Some of the weakness of RippleNet is listed below:-

1. Ripple is not completely decentralized. It is similar to traditional banking payment system which uses ILP ledger as core.

2. All coins in Ripple is premined and considered as fake Cryptocurrency in Cryptocurrency world.

3. It does not maintain users' anonymity and Governments can audit all transactions along with user details.

4. While in Blockchain miners produced new coins by processing transactions, in Ripple all coins are Premined and transaction fees are burned and paid to none.

\section{IOTA}

IOTA is a revolutionary solution for Internet of Things (IoT) and backbone of IoT technology (iota.readme.io, 2015). It is a transactional settlement and data integrity layer for the IoT and based on distributed Tangle ledger (Popov, 2014). IOTA born in 2014 and lots of new technologies which as a solution to limitation of current first and second generation Blockchain space. IOTA is third generation of Blockchain technology which provide function as the lightweight distributed ledger with scalability, quantum resistance and decentralization for all IoT devices and do not have any mining, blocks, difficulty or transaction fees (Tangleblog.com, 2015). 

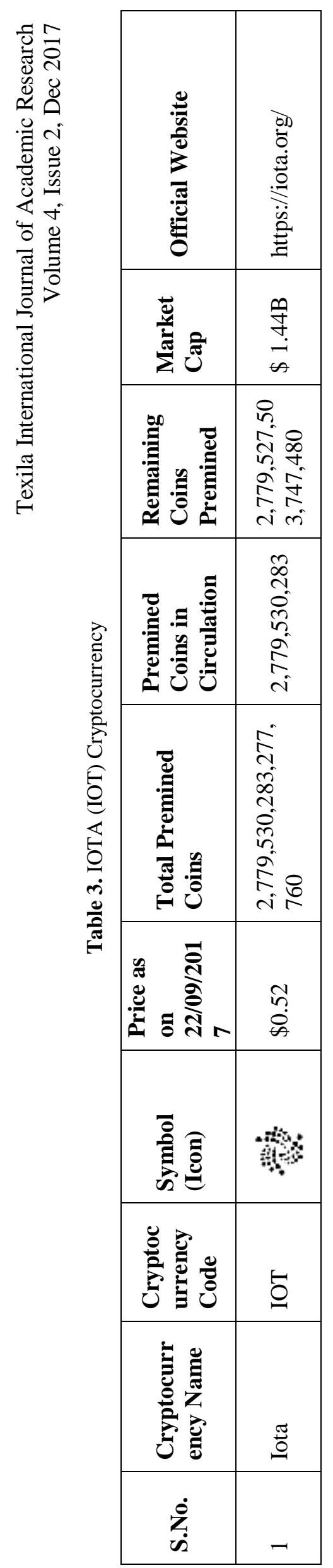
DOI: $10.21522 /$ TIJAR.2014.04.02.Art012

ISSN: $2520-3088$

IOTA is just not a Cryptocurrency but it is complete platform for IoT devices and can be used to support Micro/Nano transaction without any fees, Data transfer, Voting, Masked Messaging, anything that need distributed ledger as the Ledger of Things (LoT), financial activities as the Economy of Things (EoT).

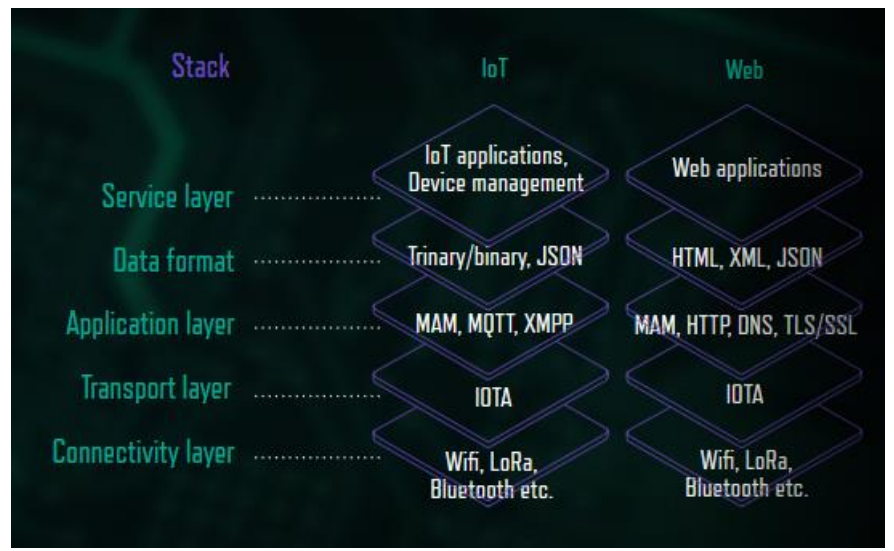

Figure 5. The IOT layers with IOTA as transport layer

The Tangle, a Directed Acyclic Graph $\left(\mathrm{DAG}^{1}\right)$ is the core of this modern Cryptocurrency which is records all transaction in IOTA. Markov Chain Monte Carlo (MCMC) algorithms select attachment sites in the tangle for just arrived transaction (Popov, The Tangle, 2017).

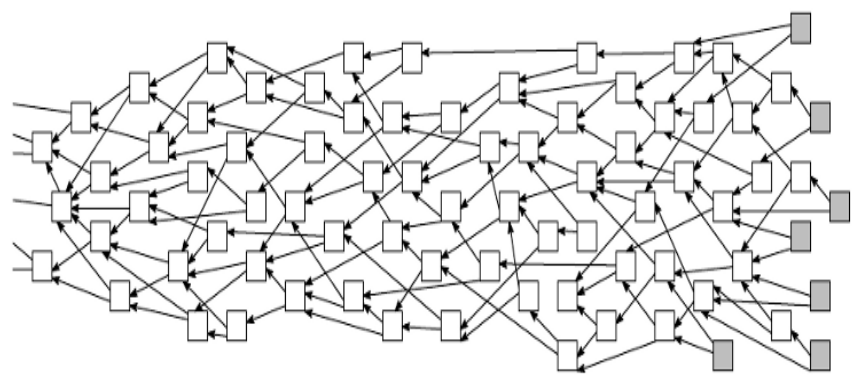

Figure 6. Directed acyclic graph (DAG)

Some of the Strength of IOTA is listed below:-

1. The biggest strength of IOTA is zero transaction fees but you have to only confirm other 2 previous transaction then after your transaction get confirmed.

2. It is blockless Blockchain technology.

3. In IOTA no need to mine coins thus no need to pay any fee to miners.

4. Internet of Things (IoT) is 4th industrial resolution and IOTA is just not support IoT but also Internet of Everything (IoE).

5. First Quantum proof Blockchain technology.

6. It supports micro-transaction and is based on Machine 2 machine economy.

7. Scaling is Infinitely.

Some of the weaknesses are listed below :-

1. It is in development phase and still evolving.

2. There could be lots of hidden bugs in IOTA implementation as it is in early phase.

3. EoT, LoT and Machine-to-Machine transactions, scale till infinity, etc. are on paper only. There are huge difficulties that need to address while implementation.

4. All coins are premined.

5. There is not concept of mining.

\footnotetext{
${ }^{1}$ See Directed Acyclic Graph (DAG) in Figure 5.
} 


\section{PeerCoin}

PeerCoin or PPCoin is first Cryptocurrency which uses Proof-of-Stake concept along with Proof-of-Work. PPCoin is a hybrid model where Proof-of-stake takes care of network security. Proof-of-stake is based on coin age and every node which is same as Bitcoin. Centrally broadcasted checkpoint mechanism is used to protect Blockchain history and transaction settlement in PPCoin (King \& Nadal, 2012). Proof-of-stake as name suggest is a proof of Cryptocurrency ownership. Coin age is used to determine proof-of-stake. Coin holding period is known as Coin Age. In order to transaction timestamp is used to facilitate the computation of coin age. Block timestamp and transaction timestamp is used to protect the computation of coin age. 


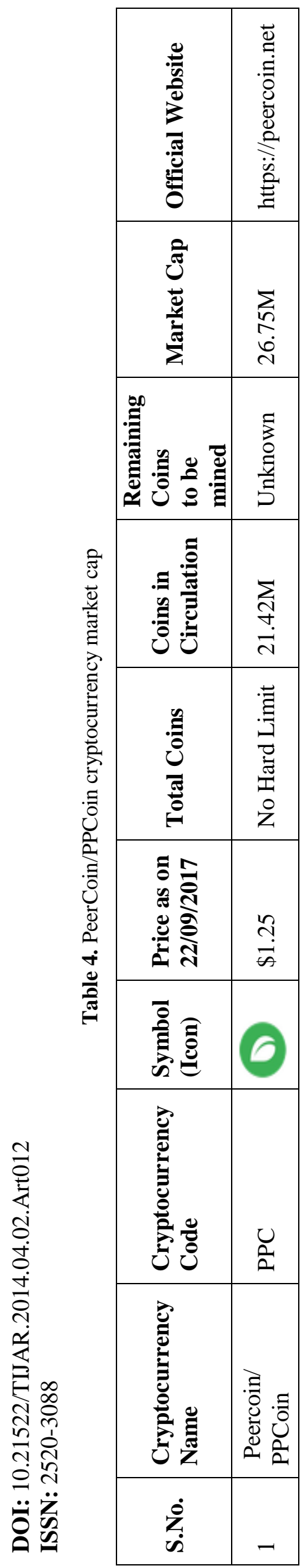


Some of the Strengths of PPCoin ${ }^{2}$ is listed below:-

1. PPCoin is based on fair distribution and are having no pre-sale of Cryptocurrency or instant mining.

2. PPCoin is the first Cryptocurrency which uses Proof-of-Stake concept to secure network along with centrally broadcasted checkpoint mechanism.

3. PPCoin is stable and secure. It is in function since 2012.

4. PPCoin is energy efficient and consume less power than other Blockchain based Cryptocurrency.

5. PPCoin is based on Bitcoin with Proof-of-stake implementation.

Some of the weaknesses of PPCoin is listed below:-

1. Being an open source community the design and protocol specification is hidden from public.

2. PPCoin algorithm uses SHA256 which can be broken in future with the help of quantum computers.

3. Lack of transparency and security is major issue in PPCoin.

4. Transaction one started cannot be reversed.

\section{Discussion and Conclusion}

There is no doubt that Cryptocurrency is addressing some of the functional issues in the current fiat currency and trying to give more control to the users as trust on governments have shaken due to mishandling of core economic issues. Cryptocurrency cannot be counterfeit and can be transacted instantly with either low or no transaction fees. Blockchain is the wonderful technology that has been invented after Internet. Proof-of-stake is another solution which shows that first generation Blockchain can improve further. Technology like RippleNet with xCurrent is a successful solution for current banking and financial institution and more than 90 organization worldwide already using Ripple based technology. IOTA is future ready technology for IoT and solid solution to power hungry Blockchain technology with zero transaction cost. However this is just a beginning Blockchain is just 2 decade old but growing rapidly. Blockchain is the future and answer to digitally driven global economy.

Various governments are having compliance and legal concern over uses of Cryptocurrency. Authorities are arguing that Cryptocurrency are being misused to illegal activities and trying to stop Cryptocurrency uses which is very unfortunate. In fact Fiat currency is being missused for this kind of activity so long and authories are failed to stop it. This is really contradictory argument and need more and open minded discussion on this subject area so that $\mathrm{t}$ can be resolved via dialog and issuing legal guidelines for compliance. Governments must also have to understand that they cannot control or stop Cryptocurrency. This is beyond their control. Cryptocurrency communities and Governments have to find out solutions for a win-win situation for all of stockholders.

\section{References}

[1].Back, A. (2002, 8 1). Hashcash- A Denial of Service Counter-Measu. Retrieved 4 25, 2017, from hashcash.org: http://www.hashcash.org/papers/hashcash.pdf.

[2].Becker, J., Breuker, D., Heide, T., Holler, J., Rauer, H. P., \& Böhme, R. (2013). Can We Afford Integrity by Proofof-Work? Scenarios Inspired by the Bitcoin Currency. The Economics of Information Security and Privacy, 135-156. doi:https://doi.org/10.1007/978-3-642-39498-0_7.

[3].Dadda, L., Macchetti, M., \& Owen, J. (2004). The design of a high speed ASIC unit for the hash function SHA-256 (384, 512). Proceedings Design, Automation and Test in Europe Conference and Exhibition (pp. 70-75). IEEE.

[4].Dai, W. (2017, 04 10). B-money. Retrieved from weidai.com: http://www.weidai.com/bmoney.txt

[5].Eastlake 3rd, D., \& Jones, P. (2001). US secure hash algorithm 1 (SHA1). (No. RFC 3174).

[6].FCIC. (2011). FINAL REPORT OF THE NATIONAL COMMISSION ON THE CAUSES OF THE FINANCIAL AND ECONOMIC CRISIS IN THE UNITED STATES. THE FINANCIAL CRISIS INQUIRY COMMISSION. Retrieved 04 05, 2017, from http://fcic-static.law.stanford.edu/cdn_media/fcic-reports/fcic_final_report_full.pdf.

[7].FCIC. (2017, 04 22). History of the commission. Retrieved from Cybercemetery. unt.edu's Archive: https://cybercemetery.unt.edu/archive/fcic/20110310173617/http://www.fcic.gov/about.

[8].Finney, H. (2005). RPOW: Reusable Proofs of Work. CodeCon.

[9].iota.readme.io. (2015). IOTA Introduction. Retrieved 05 22, 2017, from iota.readme.io: https://iota.readme.io/v1.2.0/docs.

\footnotetext{
${ }^{2}$ Market capitalization information is given in Table 4 .
} 
DOI: $10.21522 /$ TIJAR.2014.04.02.Art012

ISSN: 2520-3088

[10]. King, S., \& Nadal, S. (2012). PPCoin: Peer-to-Peer Crypto-Currency with Proof-of-Stake. Peercoin.net Whitepaper, 1-6. Retrieved 06 05, 2017, from https://peercoin.net/assets/paper/peercoin-paper.pdf.

[11]. Michael, C., Pattanayak, P., Verma, S., \& Kalyanaraman, V. (2016). Blockchain technology: Beyond bitcoin. Applied Innovation 2, (pp. 6-10).

[12]. Nakamoto, S. (2008). Bitcoin: A Peer-to-Peer Electronic Cash System. bitcoin.org. Retrieved 04 20, 2017 , from https://bitcoin.org/bitcoin.pdf.

[13]. Peck, M. (2012, 05 30). Bitcoin: The Cryptoanarchists' Answer to Cash. Retrieved from spectrum.iee.org: https://spectrum.ieee.org/computing/software/bitcoin-the-cryptoanarchists-answer-to-cash/0.

[14]. Percival, C., \& Josefsson, S. (2016, 07). The scrypt Password-Based Key Derivation Function. Internet Engineering Task Force (IETF). Retrieved 03 25, 2017, from https://tools.ietf.org/html/rfc7914.

[15]. Popov, S. (2014). The tangle. IOTA Whitepaper, 1-25. Retrieved 04 05, 2017, from iota.org: http://iota.org/IOTA_Whitepaper.pdf.

[16]. Popov, S. (2017). The Tangle. Version 1.2, 1-28. Retrieved $04 \quad 05, \quad 2017$, from https://www.docdroid.net/mWTNlgd/iota1-2.pdf.

[17]. Ripple.com. (N.D.). Product Overview. Retrieved 05 13, 2017, from Ripple.com: https://ripple.com/files/ripple_product_overview.pdf.

[18]. Ripple.com. (N.D.). Solution Overview. Retrieved 05 04, 2017, from ripple.com: https://ripple.com/files/ripple_solutions_guide.pdf.

[19]. Szabo, N. (2008, 12 07). Bit gold. Retrieved 04 02, 2017, from unenumerated.blogspot.com: https://unenumerated.blogspot.com/2005/12/bit-gold.html.

[20]. Tangleblog.com. (2015). IOTA- what is it, who is it? Retrieved 05 04, 2017, from tangleblog.com: http://www.tangleblog.com/what-is-iota-what-is-the-tangle/.

[21]. Thomas, S., \& Schwartz, E. (2015). A Protocol for Interledger Payments. Retrieved 05 03, 2017, from interledger.org: https://interledger.org/interledger.pdf.

[22]. Williams, M. (2010). Uncontrolled Risk. McGraw-Hill Education. 